\title{
Stability and Vulnerability of Bird Flocking Behaviour: A Mathematical Analysis
}

\author{
Bayu Erfianto ${ }^{1 *}$, Intan Muchtadi-Alamsyah ${ }^{2}$ \\ ${ }^{1}$ Department of Information Technology, Telkom University, Bandung, Indonesia \\ ${ }^{2}$ Algebra Research Group, Faculty of Mathematics and Natural Sciences, Institut Teknologi Bandung, Bandung, Indonesia
}

ARTICLE INFO

Article history:

Received February 10, 2017

Received in revised form September 20, 2018

Accepted January 30, 2019

\section{KEYWORDS:}

flocking,

communication network,

stable,

vulnerable

\begin{abstract}
Given a large number of birds in the flock, we mathematically investigate the mechanism the birds move in a collective behavior. We assume that each bird is able to know its position and velocity of other birds within a radius of communication. Thus, to be able to fly in the flock, a bird has to adjust its position and velocity according to his neighbors. For this purpose, first of all, we analyze how the connectedness of the bird interaction network affects the cohesion of the stable bird flock. We further analyze a condition when the flock is vulnerable, which is mathematically indicated by means of the presence of an articulation point in bird communication network.
\end{abstract}

\section{Introduction}

Our object of study is the flocking behavior during which the birds moving in a group and make whole decisions of flight autonomously in the absence of leader. Within the last few years, many researchers pay attention to mechanism of how birds flock rather than why. During the flock, actually more eyes of birds may increase the opportunities to find food, such as in a foraging, and improve the chances of detecting a predator as well. When a predator approaches the flock, all the individual birds react to move toward the safest position in order to reduce the chances of being captured.

Nowadays, in engineering, flocking can be seen as one of the simple collective behavior of individuals in a group. This collective behavior model is also well known as multi-agent systems, which attracts lots of attention with respect to engineering applications, for instance in formation control of robots and unmanned aerial vehicles (Olfati-Saber 2006; Tanner et al. 2007; Yang et al. 2010; Erfianto and Bambang 2012; Williams and Sukhatme 2013). Very recently, the synthesis of dynamics of flocking model in the area of cooperative control have also been done by Viscek (Vicsek and Zafeiris 2012), and the JusthKrishnaprasad (Galloway et al. 2013). The agent is

* Corresponding Author

E-mail Address: erfianto@telkomuniversity.ac.id modeled as a particle, which moves with a certain velocity vector in the planar area $\mathrm{R}^{n}$.

Motivated by Reynolds's flocking model (Reynolds 1987), lots of distributed algorithms have been developed for in term of cooperative control, for example in (Tanner et al. 2003). This model focus on cohesion of motion for a group of interconnected dynamic system. The stability analysis as well as the convergence proofs for the group cohesion have also been examined, such as to synchronize all individuals to reach the same velocity vectors based on the algebraic connectivity properties of the communication network (Liu and Passino 2004; Gazi and Passino 2011; Zavlanos et al. 2011; Sun 2013). Since then, researchers use graph theoretic approach that provides the fundamental tools for design and analysis of coordinated motion, especially inspired by dynamics of bird flock.

Recently, (Ghedini et al. 2015) investigated the robustness of network of multi-agent systems from the other point of view. They propose a mechanism for detecting the probability of an agent being disconnected. In their model, each individual agent is able to detect critical network topology and mitigate an agent in order to move to a new position to improve connectivity measure. A node that is potentially to be lost will cause disconnection of the whole communication network. Mathematically, this node is then defined as articulation points. Regarding this 
property, the graph which has no articulation points is called biconnected graph (Ahmadi and Stone 2006).

In this paper, we address how can a bird, which is represented by an individual autonomous agent, move towards a stable flocking behavior, which means that the inter-individual distance is bounded by communication constraint, no collision among birds, and the flocking condition is achieved when individuals reach the same velocity vector asymptotically. This necessary condition will be examined in mathematical model. In the second part of this paper we address how can a bird detects a failure of individual bird, which will cause the flocking behavior disconnected.

\section{Materials and Methods}

In this section, some definitions on algebraic graph theory as the underlying theory of bird communication network will be briefly presented. We also recall some works on common flocking model in engineering.

\subsection{Graph Theory}

Suppose a bird makes communication over a hundred of individuals using sound and visual communication, and this communication pattern is then called flocking topology. We model flocking topology as undirected graph $\mathrm{G}=(\mathrm{V}, \mathrm{E})$, where $\mathrm{V}=\left\{v_{1}, \ldots, v_{n}\right\}$ is set of nodes (individual bird), and $\mathrm{E}=\left\{\left(v_{i}, v_{j}\right) \mid i, j \in\{1,2, \ldots, n\}\right\}$ is set of edges (interaction among individual bird). Assume $G$ is simple that is no self-loop $\left(v_{i}, v_{i}\right) \notin E$ and no multiple edge exist between $\left(v_{i}, v_{j}\right)$. A graph $G$ is regular graph if every node has exactly $k$ communication edges, and we say that every node has degree $k$. The flocking topology $\mathrm{G}$ is then encoded by an $n \times n$ adjacency matrix, where $a_{i j}>0$ if $(i, j) \in \mathrm{E}$, and $a_{i j}>0$ otherwise. The Laplacian matrix of a graph $\mathrm{G}$ is defined as $L=$ $D A$, where $A$ is adjacency matrix and $D$ is degree of matrix $A$. The element of Laplacian matrix $L$ is denoted by $l i$. Due to the characteristic of Laplacian matrix, it can be seen that $L$ has positive real eigenvalues for undirected graph G (Godsil et al. 2001). Denote $\lambda_{i}(L)$ as the $i$-th eigenvalue, and $v_{i}(L)$ as an associated right eigenvector of Laplacian matrix $L$. Because of the symmetry of $L$, those the eigenvalues are real, and can be ordered as $0=\lambda_{1}(L) \leq \lambda_{2}(L) \leq \ldots \leq \lambda_{n}(L)$. Fiedler (Fiedler 1973) denotes that the connectivity measure of a communication graph $\mathrm{G}$, or flocking topology in our case, is denoted by $\lambda_{2}(L)$, which is well known as algebraic connectivity. Thus, it means that, the strong the graph connected the larger the algebraic connectivity it has. Thus, the topology of graph $\mathrm{G}$ is said to be robust.

\subsection{Flocking: An Engineering Perspective}

To have social interactions among group member during flock, thus it means that a bird has to communicate each other over a hundred of individuals, for instance using sound and visual communication. Meanwhile biologists investigated that many birds use the limited range of communication to form a flocking formation (Weiss et al. 2014). The other model also came from computer scientist and mathematicians. They study the collective behavior of bird flock is to model and study them using computer simulations. To the best of our know-ledge, the models are based on three distinct features of collective movement i.e., alignment of velocities, attraction, and short-range repulsion (Reynolds 1987).

Consider the motion of individual bird in twodimensional space $\mathrm{R}^{2}$. Let $q_{i}=\left[\begin{array}{ll}x_{i} & y_{i}\end{array}\right]^{\mathrm{T}} \in \mathrm{R}^{2}$ be the vector position of individual bird $i$. Suppose all interaction among bird is assumed as undirected graph, that is the adjacency matrix element $a_{i j}=a_{j i}$. The neighborhood of bird agent $i$ is bounded by $\mathrm{N}_{i}$ $=\left\{j \in \mathrm{V} \mid q_{i j} \leq d\right\}$, where $q_{i j}$ the Euclidean distance between bird $i$ and $j$, and $d$ is maximum distance that enables paired-interaction between birds. The illustration of this model is depicted in Figure 1.

From engineering point of view, there are at least three multi-agent coordination and control problems (Gazi and Passino 2011), and bird flocking is similar to aggregation problem. Suppose there are $N$ individual agents with the following position states $q_{i j} \in \mathrm{R}^{2}, i=1$, .., $N$. Let $\bar{x}$ be the centroid of group, i.e. the average position of aggregation point, which is denoted as $\bar{x}=\frac{1}{N} \sum_{i=1}^{N} q_{i}$. Each agent requires internal force (in control theory it is a control input) $u_{i}, i=1, \ldots, N$ to drive the agent in order to stay close in a group w.r.t. the centroid. Thus, the aggregation problem is how to model $u_{i}$ such that as $t \rightarrow \infty$ for all agents, we have $\lim \left\|q_{i}-\bar{x}\right\| \leq d_{c}$, where $d_{c}$ is maximum distance to the centroid. Motion of flocking is mathematically modeled as attractive and repulsive function between two individual birds, which is comprehensively discussed in (Gazi and Passino 2004):

$$
u_{i}=-\sum_{j \in N_{i}} \nabla q_{i}\left[\phi_{a}\left(\left\|q_{i-} q_{j}\right\|\right)-\phi_{r}\left(\left\|q_{i-} q_{j}\right\|\right)\right]
$$




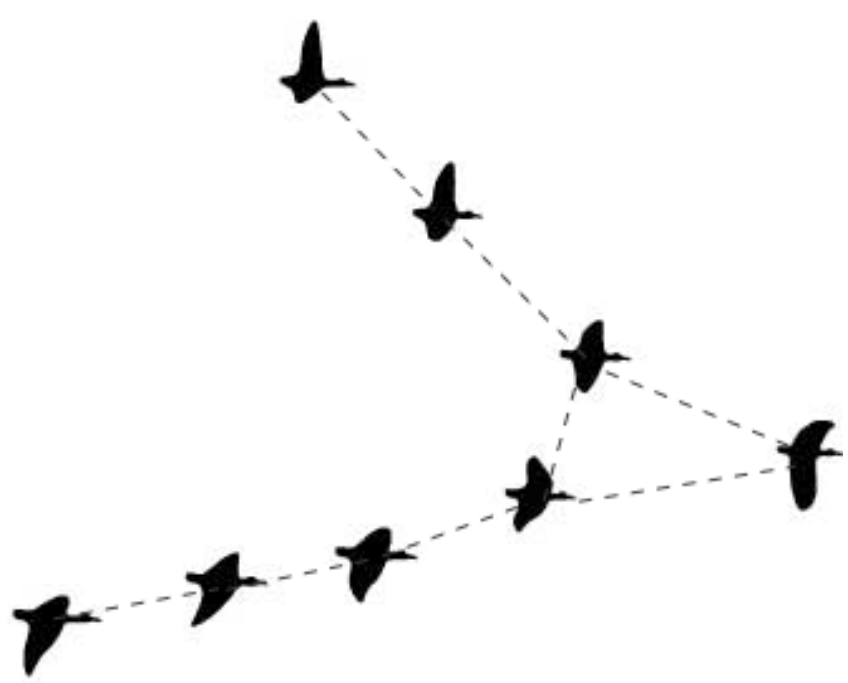

Figure 1. Bird Flocking with interaction model represented as undirected graph

Physically, $q_{i} \in \mathrm{R}^{2}$ is the position of bird $\mathrm{i}$ in a twodimension coordinate frame. The notation of $\nabla_{q_{i}} \phi_{a}\left(\left\|q_{i}-q_{j}\right\|\right)$ is to model a virtual force between two individual bird. This virtual force is intended to attract the neighboring bird as close as possible when it is too far away, while the notation of $\nabla_{q_{i}} \phi_{r}\left(\left\|_{q_{i}}-q_{j}\right\|\right)$ is to model virtual repulsive force. The repulsive virtual force is intended to repel the neighboring bird when it is too close to each other, hence the collision can be avoided.

\section{Results}

The problem in this paper is how to model stable bird flocking behavior. For this purpose, we define stable flocking mechanism. Group of birds is said to achieve stable flock, when (1) inter-bird distance is stable such that flock is collision free, (2) all birds approach the same velocity vector asymptotically, and (3) the flocking topology is always connected, which is mathematically defined as:

$$
\left\{\begin{array}{c}
0<\left\|q_{i}-q_{j}\right\| \leq R \\
v_{i}=v_{j} \\
\lambda_{2}>0
\end{array}\right\}
$$

where $\lambda_{2}$ is the second smallest eigenvalue of Laplacian matrix $L$ of bird communication topology and $R$ is maximum distance of interaction between birds. We use mathematical approach in (Erfianto et al. 2016) to model the bird flocking behavior that consists of inter-bird attraction-repulsion force, group centroid function from Equation 1, and bird velocity matching, which is defined in the following equation.

$$
u_{i}=-\sum_{j \in N_{i}} \nabla q_{i} \varphi_{a}\left(q_{i j}\right)-\sum_{j \in N_{i}}\left\|q_{i}-\bar{x}\right\|+\sum_{j \in N_{i}} a_{i j}\left(p_{i}-p_{j}\right)
$$

Our model should conform to criteria in Equation 2 as necessary condition to stable flocking behavior. We use Lyapunov technique to check the stability of our flocking behavior model. Let all terms in Equation 3 is denoted as Pi and let $\mathrm{V}$ be Lyapunov candidate function such that:

$$
V=\sum_{j \in N_{i}}\left[P_{i}\left(q_{i, j}\right)+\frac{1}{2} \sum_{j \in N_{i}}\left[v_{j}-v_{i}\right]\right]
$$

To prove the stability of our flocking model, the same technique as described in (Erfianto et al. 2016) is used. The derivative of Equation 4 is obtained as:

$$
\dot{V}=-v^{T}\left(L \otimes I_{N}\right) v \leq 0
$$

where $I_{N}$ is identity matrix, and $v$ is matrix of velocity vector. Regarding Lyapunov stability analysis, as $t>0$, if the derivative of Lyapunov candidate $\leq 0$, it is asymptotically stable. This physically means that the birds in our model finally could reach the same velocity and no collision occurs as the necessary condition in Equation 2. Further, the connectedness of the flocking topology, which is represented in Laplacian matrix, directly affects the cohesion in the stable flock, such that $\lambda_{2}(L)>0$ for all $t>0$.

The result of simulation of Equation 3 can be seen in Figure 2 and 3 that show the stable trajectory of bird flocking. The stability of flocking trajectory is also indicated by the second smallest eigenvalue, which is depicted by the plot of the second smallest eigenvalue in Figure 2 and 3.

\section{Discussion}

In this subsection, we use Theorem 1 and 2 in (Zareh et al. 2016) to check the vulnerability of our results in Equation 3 to 5 of bird flocking configuration network. Theorem 1 in (Zareh et al. 2016) proves the eigenvalue of perturbed of Laplacian matrix, while Theorem 2 in (Zareh et al. 2016) is used to determine whether a node $i$ is an articulation point or not by observing the minimum of third smallest eigenvalue of perturbed Laplacian matrix. Physically, when a bird in flock moves away from its neighboring birds, it affects to the topology of flocking formation, in other word flocking 


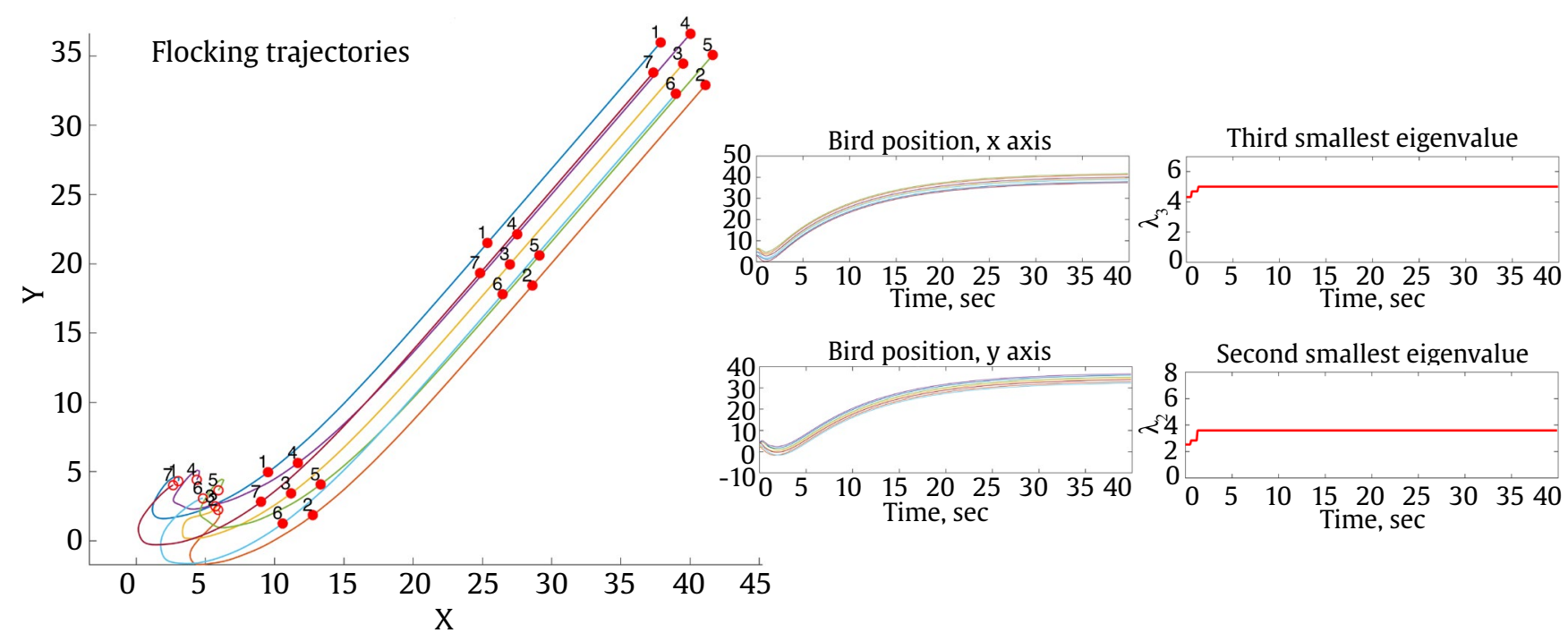

Figure 2. Simulation of bird flocking. Left: trajectory of bird flock from initial position to reach a destination. Right: plot of bird position in 2 dimensions, third and second smallest eigenvalue of bird flocking formation

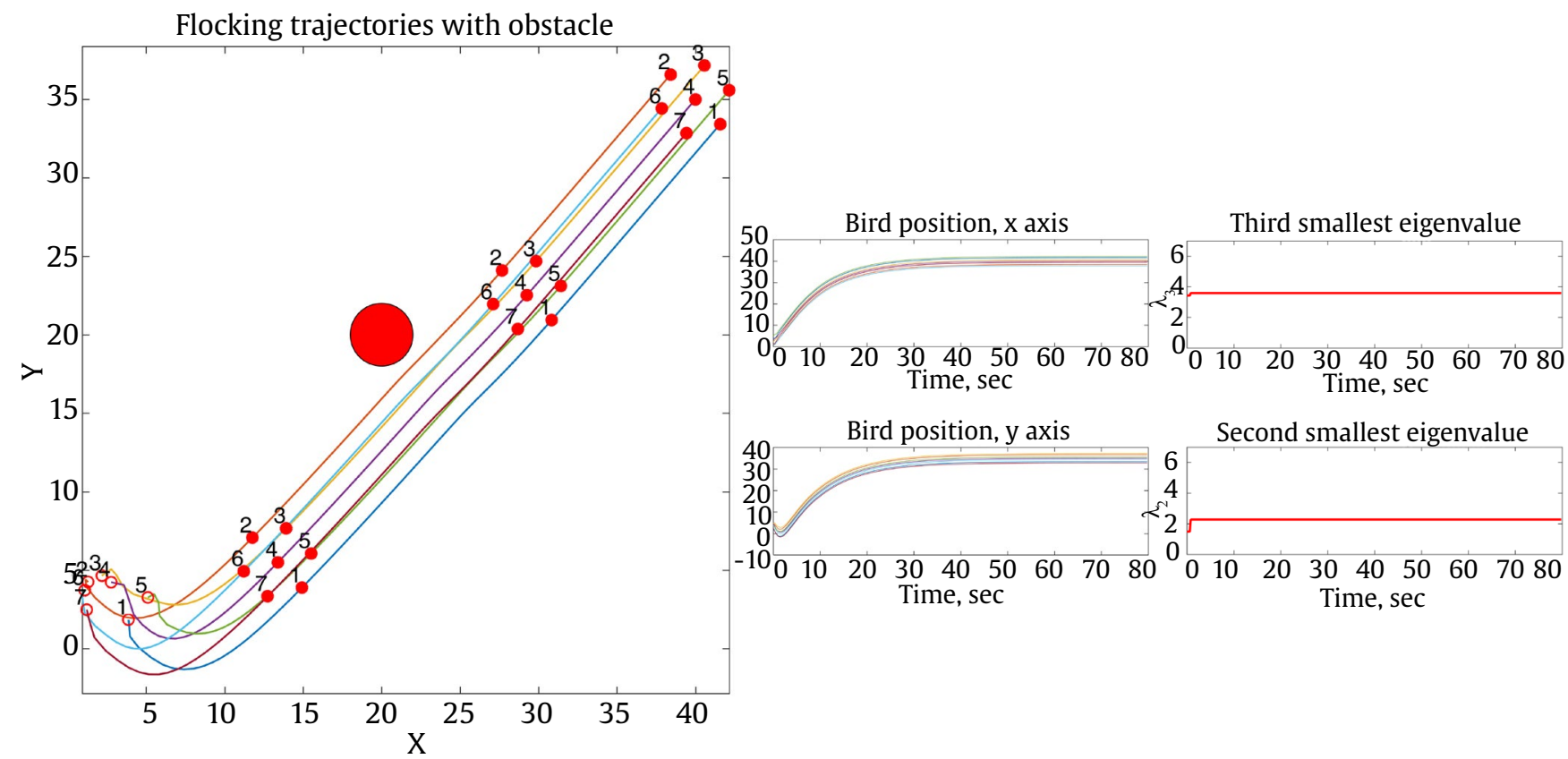

Figure 3. Simulation of bird flocking with obstacle. Left: trajectory of bird flock from initial position to reach a destination. Right: plot of birds position in 2 dimension, third and second smallest eigenvalue of bird flocking formation

formation is perturbed. Thus, by using algebraic graph theory, it can be analyzed whether this moving bird is an articulation point or not that perturb the flocking formation. For instance, a predator can be considered as the external disturbance that may perturb the flocking formation.

During flock, the flocking network or flocking topology may change due to presence of predators. Therefore, the previous Laplacian matrix in Equation 5 is perturbed by the external disturbance. The idea is how we can use perturbed Laplacian matrix to detect whether the flocking topology is vulnerable or not. If vulnerable, this will affect to the separation of the existing flocking topology.

To model the vulnerable of flocking topology, we can use the definition of articulation point in a graph $\mathrm{G}$ as described in (Ahmadi and Stone 2006). A bird that is modeled as a node $\mathrm{i} \in \mathrm{V}$ of a connected flocking topology $\mathrm{G}$ is called an articulation point if a reduced graph $G^{R} \subset G$ is not connected. Thus, a connected graph 
$\mathrm{G}$ is called biconnected if $\mathrm{G}$ contains no articulation point.

Adopting the above concept into flocking behavior, we actually can detect whether a bird is an articulation point or not with respect to flocking topology.

1. First of all, a bird may check the degree of interaction among the other birds, or mathematically equals to the degree of connectivity $d_{i}$. If the $d_{i}$ is small enough, then continue to step 2 .

2. Reduce the graph $\mathrm{G}$ into $\mathrm{G}_{i}^{R}$ that contains node $i$ as a suspected articula-tion point.

3. Zareh in (Zareh et al. 2016) introduced perturbed Laplacian matrix $\mathrm{L}_{i}(\mathrm{~s})$ obtained from perturbed adjacency matrix in reduced graph $G^{R}$. Thus, we can directly use Theorem 2 in (Zareh et al. 2016) to check whether the third smallest eigenvalue of reduced Laplacian matrix $\mathrm{L}_{i}(\mathrm{~s})$ satisfies:

$$
\lambda_{3}\left(\mathcal{L}_{i}(\epsilon)\right)>\epsilon \sqrt{N}\left(\sum_{j=1}^{N} a_{i j}^{2}\right)^{\frac{1}{2}}
$$

where $s \in R$ is real scalar value.

If Equation(6) holds, then the bird in reduced flocking topology $G_{i}$ meets the sufficient conditions for being nonarticulation point. Otherwise, if bird $i$ is an articulation point, then the whole flocking topology is vulnerable though the robustness measure $\lambda_{2}(L)>0$ of the graph $G$ also holds for $t>0$. If there exist an articulation point, this physically means that a flocking topology may be separated easily, and a bird acts as an articulation point is easily being captured by a predator. To proof the concept of our approach, we also provide a simulation of flocking behavior, which is depicted in 2 and 3. It can be drawn from the simulation that the flocking model is stable, which is indicated by $0<\lambda_{2}(L)<\lambda_{3}(L)$. To check the existence of articulation, point that make the flocking formation dismiss, from Theorem 2 in (Zareh et al. 2016), it can be concluded that since the perturbed flocking formation causes perturbed $\lambda_{2}(L)<\lambda_{3}(L)$, then the flocking formation contains no articulation point. Thus, the flocking formation is not vulnerable.

The result of simulation of Equation 6 is depicted by the plot of the third smallest eigenvalue in Figure 2 and 3.

\section{Conclusion}

We present the analytical model of bird flocking behavior using algebraic graph and control engineering theory. Regarding Lyapunov stability theory, which is commonly used in control engineering, our model is asymptotically stable. By observing the second smallest eigenvalue of Laplacian matrix of flocking topology, we can also observe the robustness of bird flocking behavior. However, since the second smallest eigenvalue of Laplacian matrix only measure the connectedness, the vulnerable of a flocking topology cannot be drawn from this measure. The use of perturbed Laplacian matrix of reduced flocking topology represents the presence of disturbance on the flocking behavior, and the third smallest eigenvalue of perturbed Laplacian matrix can be directly used to measure the vulnerability of the whole flocking formation.

\section{References}

Ahmadi M, Stone P. 2006. A distributed biconnectivity check. In: Gini M, Voyles R (Eds.). Distributed Autonomous Robotic Systems 7. Tokyo: Springer. pp. 1-10.

Erfianto B, Bambang R. 2012. Swarm robot flocking assisted by explicit communication. In: Proceedings International Conference on Intelligent Unmanned System. Singapore: SIM University. pp. 233-239.

Erfianto B et al. 2016. Design of connectivity preserving flocking using control lyapunov function. Journal of Robotics Hindawi 2016:1-12.

Fiedler M. 1973. Algebraic connectivity of graphs. Czechoslovak Mathematical Journal 23:298-305.

Galloway KS et al. 2013. Symmetry and reduction in collectives: cyclic pursuit strategies. In: Proceedings of The Royal Society A: Mathematical, Physical and Engineering Sciences. UK: The Royal Society. pp. 1-23.

Gazi V, Passino KM. 2004. A class of attractions/repulsion functions for stable swarm aggregations. International Journal of Control 77:1569-1579.

Gazi V, Passino KM. 2011. Swarm Stability and Optimization. Heidelberg: Springer.

Ghedini C et al. 2015. Improving robustness in multi-robot networks. IFAC-PapersOnLine 48:63-68.

Godsil C et al. 2001. Algebraic Graph Theory Vol. 8. New York: Springer.

Liu Y, Passino KM. 2004. Stable social foraging swarms in a noisy environment. IEEE Transaction on Automatic Control 49:30-44.

Olfati-Saber R. 2006. Flocking for multi-agent dynamic systems: algorithms and theory. IEEE Transactions on Automatic Control 51:401-420.

Reynolds CW. 1987. Flocks, herds and schools: a distributed behavioral model. In: Proceedings of the $14^{\text {th }}$ annual conference on Computer graphics and interactive techniques. New York: Published by ACM SIGGRAPH Computer Graphics. pp. 25-34.

Sun Y. 2013. Stability analysis of flocking for multi-agent dynamic systems. Non- linear Analysis: Real World Applications 14:1075-1081.

Tanner HG et al. 2003. Stable flocking of mobile agent part II: dynamic topology. In: Proceedings of the $42^{\text {nd }}$ IEEE Conference on Decision and Control. Maui: Published by IEEE. pp. 2016-2021. 
Tanner HG et al. 2007. Flocking in fixed and switching networks. IEEE Transactions on Automatic Control 52:863-868.

Vicsek T, Zafeiris A. 2012. Collective motion. Physics Reports 517:71-140.

Weiss M et al. 2014. The use of network analysis to study complex animal communication systems: a study on nightingale song. Proceedings of the Royal Society $B$ 281:1-9.

Williams RK, Sukhatme GS. 2013. Topology-constrained flocking in locally interacting mobile networks. In: Proceedings International Conference on Robotics and Automation. Karlsruhe: IEEE. pp. 2002-2007.
Yang P et al. 2010. Decentralized estimation and control of graph connectivity for mobile sensor networks. Automatica 46:390-396.

Zavlanos MM et al. 2011. Graph-theoretic connectivity control of mobile robot networks. Proceedings of the IEEE 99:1525-1540.

Zareh M et al. 2016. Decentralized biconnectivity conditions in multi-robot systems. In: Proceedings Decision and Control, IEEE Conference on. Las Vegas: IEEE. pp. 99-104.

Zareh M et al. 2016. Enforcing biconnectivity in multi-robot systems, In: Proceedings Decision and Control, IEEE Conference on. Las Vegas: IEEE. pp. 1800-1805. 